\title{
Full-Wave Analysis of the Image Hybrid Dielectric/HTS Resonator
}

\author{
Carles Sans, Juan M. O'Callaghan, David Sancho, Rafael Pous, Member, IEEE, \\ Josep Fontcuberta, Ji-Fuh Liang, and Guo-Chun Liang, Senior Member, IEEE
}

\begin{abstract}
An analysis of the image hybrid dielectric/hightemperature superconductor (HTS) resonator is carried out. A full-wave radial mode-matching method is used to obtain the electromagnetic fields inside the resonator for single $\mathbf{T E}_{01}$ and dual $\mathbf{H E}_{11}$ modes. Measured resonant frequencies and quality factors of these modes are compared with numerical results of the analysis. The resonator power-handling capability is estimated from the field at the surface of the HTS film, assuming a certain value for the critical field of the HTS film.
\end{abstract}

Index Terms-Dielectric resonator filters, electromagnetic analysis, superconducting filters, superconducting resonators.

\section{INTRODUCTION}

$\mathbf{H}$ IGH-POWER performance of high-temperature superconductor (HTS) communication filters is limited by the critical current of HTS films. High current densities due to edge effects in transmission-line-based filters prevent their utilization for high-power applications. Recently, some interest has been put in the new hybrid dielectric/HTS resonator as a possible resonator structure for high-power HTS filters [1]-[3]. This resonator structure provides small size, high quality factor, and good thermal stability, allowing the realization of reduced size hybrid dielectric/HTS dual-mode filters for high-power applications.

Optimal performance of these filters requires proper design of the resonators. As a previous step to the design, this work presents the numerical analysis of the hybrid dielectric/HTS resonator. From this analysis, features that are essential to filter performance like resonant frequencies, quality factors, and power-handling capability are calculated. The numerical results are compared with measured data.

Manuscript received February 3, 1997; revised August 22, 1997. This work has been funded by CICYT through Grants MAT 95-1038-C02-02 and MAT 94-1024-C02-01, by CIRIT-SGR95-528, by CSIC through a doctoral scholarship for one of C. Sans, and by NRL through Contract N00014-94-C2106.

C. Sans is with the Universitat Politécnica de Catalunya, Campus NordD3, Barcelona 08034, Spain and the ICMAB-CSIC, Campus UAB, Bellaterra 08319, Spain.

J. M. O'Callaghan and R. Pous are with the Universitat Politécnica de Catalunya, Campus Nord-D3, Barcelona 08034, Spain.

D. Sancho was with the Universitat Politécnica de Catalunya, Campus NordD3, Barcelona 08034, Spain. He is now with Telefónica Sistemas, Barcelona, Spain.

J. Fontcuberta is with the ICMAB-CSIC, Campus UAB, Bellaterra 08319, Spain.

J.-F. Liang and G.-C. Liang are with the Conductus, Inc., Sunnyvale, CA 94086 USA.

Publisher Item Identifier S 1051-8223(97)08812-X.

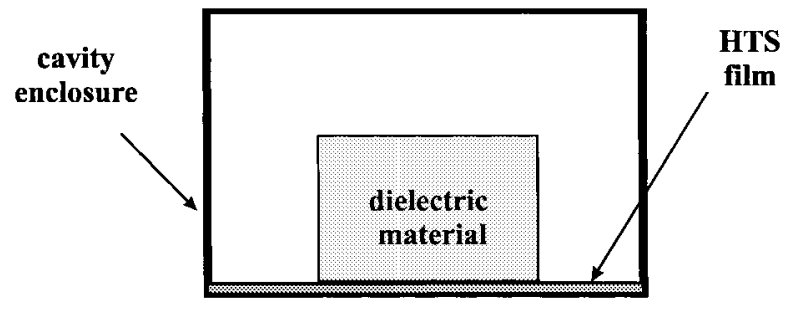

Fig. 1. Hybrid dielectric/HTS resonator. The whole structure has cylindrical symmetry.

Many methods have been used in the past to analyze dielectric resonators. In this work a full-wave radial modematching method is used which-for the geometry considered-provides low computational cost with high accuracy.

\section{Hybrid Dielectric/High-TeMPERATURE SUPERCONDUCTOR RESONATOR}

This resonator structure (Fig. 1) was first proposed in [1]. It consists of a dielectric resonator on top of an HTS thin film. The HTS film behaves as a perfect electric conductor and does not modify the fields of $\mathrm{TE}_{01} / \mathrm{HE}_{11}$ modes, since they do not have a tangential electric field on the surface. As argued in [2], the resonator is equivalent to the symmetric double size $\mathrm{HEE}_{11}$ resonator in suspended configuration.

There are several advantages of this resonator over a conventional room temperature suspended resonator: size reduction by one half, low losses of dielectric material at cryogenic temperatures, and very good thermal stability. In a normal metallic housing there would be no interest in such a structure because losses in the bottom plate of the cavity would be too high. However, using an HTS film as the cavity bottom plate does not degrade the quality factor and makes the resonator interesting for practical filters. The idea has been successfully implemented in [2] to develop a dual-mode image hybrid dielectric/HTS filter.

\section{FULL-WAVE ANALYSIS: Radial Mode-Matching Method}

The method of analysis is similar to the one in [4] and [5]. The resonator structure (Fig. 1) has cylindrical symmetry, and a two-dimensional (2-D) analysis can be done.

The software developed can analyze up to three different radial regions, each one divided into three different layers 


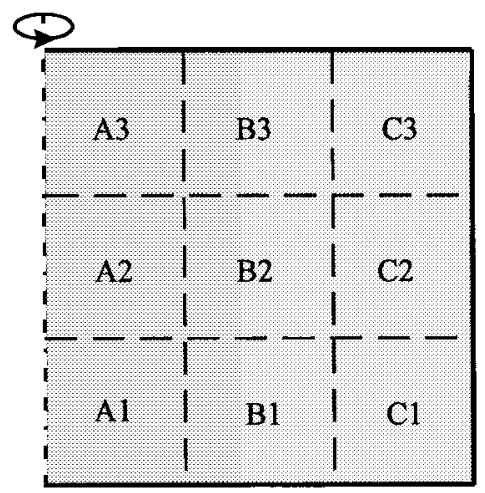

Fig. 2. One half of the resonator section. Radial regions A, B, and C.

(Fig. 2). The dielectric constant in each region and layer can be set independently, and the whole volume analyzed is assumed to be enclosed by conducting walls. The extension of this case to a higher number of radial regions or layers is straightforward. In the radial mode-matching method, fields in every radial region are expressed as an infinite series of particular solutions of the wave equation in cylindrical coordinates

$$
\left\{\frac{1}{r} \frac{\partial}{\partial r}\left\{r \frac{\partial}{\partial r}\right\}+\frac{1}{r^{2}} \frac{\partial^{2}}{\partial \phi^{2}}+\frac{\partial^{2}}{\partial z^{2}}+k^{2}\right\} \Psi=0 .
$$

Azimuthal and axial eigenfunctions are harmonic functions while radial eigenfunctions are Bessel functions. The eigenvalues of each radial region are found by solving a pair of transcendental equations related to the three-layer problem. In the geometry considered (Fig. 2), axial behavior is governed by harmonic functions (1). The radial behavior of the fields, however, is governed by Bessel functions which must be chosen appropriately to avoid singularity in the axis (region A), fulfill boundary conditions on the external walls (region C), or provide continuity between adjacent regions (region B).

After solving for the eigenvalues of TM/TE modes, and the appropriate Bessel functions for every field component are chosen; an expression of the fields in every radial region can be written in the form of an infinite series of simple TM/TE modes.

A system of equations is then found by forcing continuity conditions of the tangential fields between radial regions $\mathrm{A}$ and $\mathrm{B}$, and $\mathrm{B}$ and $\mathrm{C}$. Then, by following a Galerkin method with appropriate inner products that have orthogonality properties, we can reduce the number of equations and solve for mode expansion coefficients in region B. Mode expansion coefficients in regions $\mathrm{A}$ and $\mathrm{C}$ are obtained from coefficients in region B. Accuracy in computed fields is determined by the number of modes considered in the series expansion of the fields. The higher the accuracy, the higher the required computational cost. Moreover, there is a compromise between flexibility of the structure and computational cost. The higher the number of radial regions and layers considered, the higher the flexibility. Therefore, there is a compromise that leads to some optimum parameters for the computations. In most cases, 12 modes, three radial regions, and three layers in each radial region provide enough accuracy and flexibility. In fact, in our

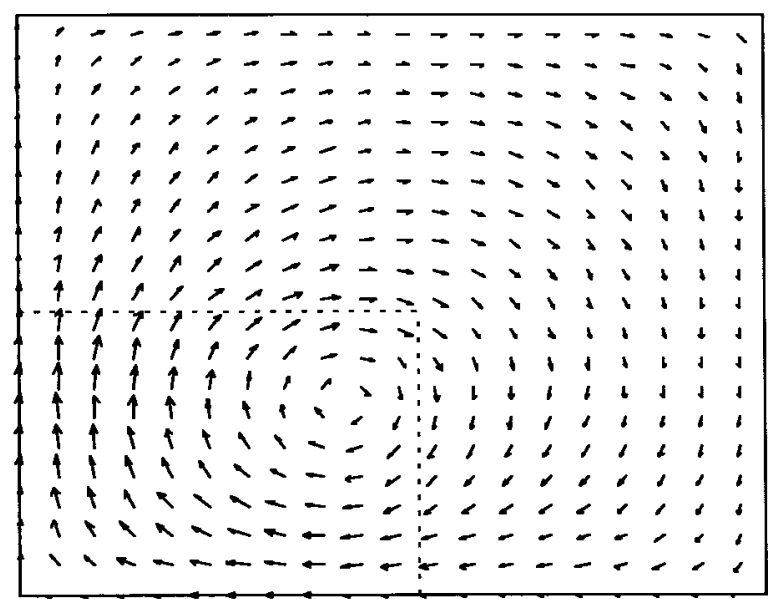

(a)

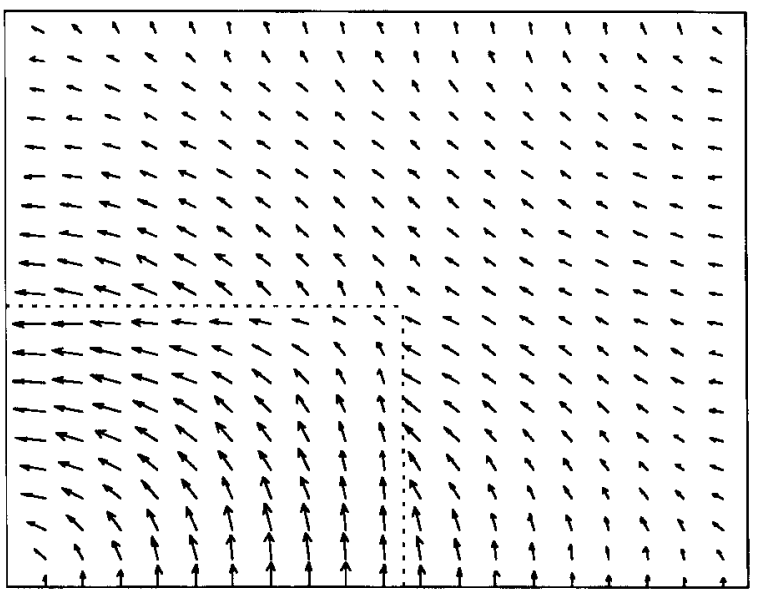

(b)

Fig. 3. (a) $\mathrm{TE}_{01}$ mode magnetic field in one half of the resonator section. Vertical axis corresponds to the axial dimension whereas longitudinal axis corresponds to the radial dimension. Dotted lines outline the volume occupied by the dielectric. (b) $\mathrm{HE}_{11}$ mode electric field in one half of the resonator section. Vertical axis corresponds to the axial dimension whereas longitudinal axis corresponds to the radial dimension. Dotted lines outline the volume occupied by the dielectric.

case, two radial regions with two layers each are enough. The inclusion of an insulating post on top of the dielectric material (which, in practice, is necessary to hold it in place and avoid air gaps between the dielectric and the HTS) can be easily taken into account in the numerical analysis by adding an extra radial region.

\section{FIELD AND CURRENT Distribution}

The method of analysis computes the field distribution of any resonant mode in the resonator. In Fig. 3, the fields in a transverse cut of the resonator for both $\mathrm{TE}_{01}$ and $\mathrm{HE}_{11}$ modes are plotted. Field intensity is much higher inside the dielectric material. Thus, if resonator design is appropriate, the overall loss of the device is dominated by dielectric losses.

Once the field distribution in the cavity is known, surface current density on the HTS film is calculated from the 


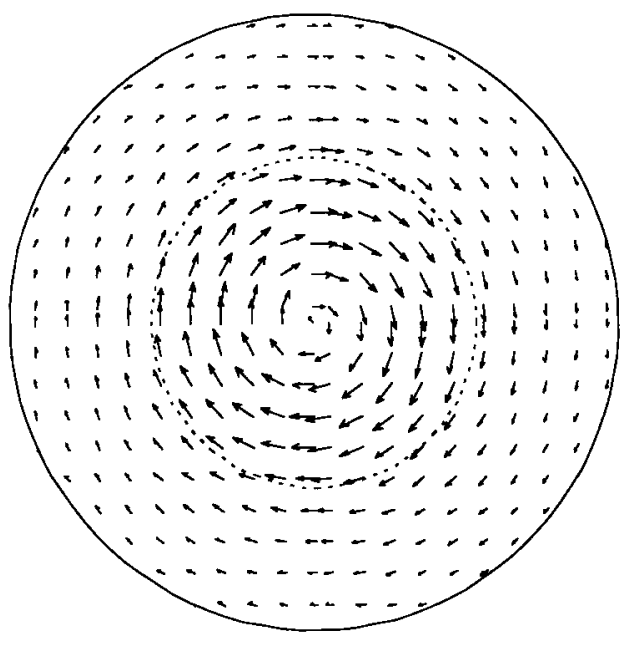

(a)

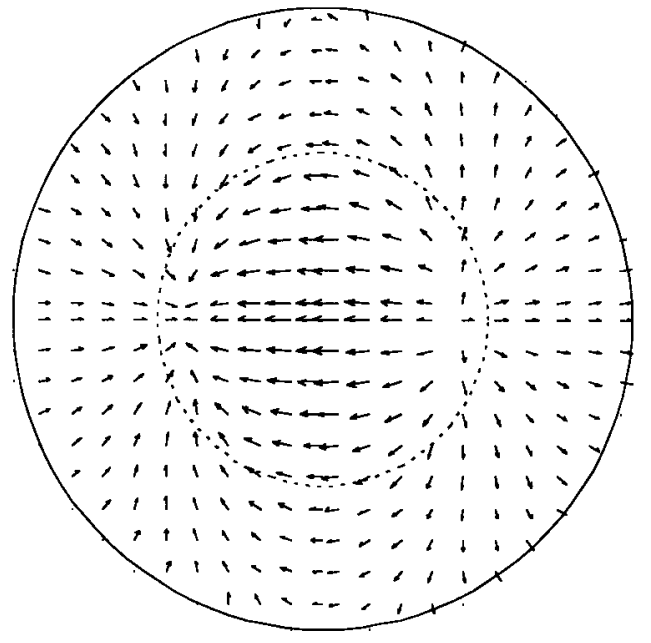

(b)

Fig. 4. (a) $\mathrm{TE}_{01}$ mode current distribution on the HTS thin film. The curren is circumferential and there is no radial current. Dotted lines outline the volume occupied by the dielectric. (b) $\mathrm{HE}_{11}$ mode current distribution on the HTS thin film. Radial currents at the edge of the HTS film are important. Dotted lines outline the volume occupied by the dielectric.

tangential components of the magnetic field

$$
\mathbf{J}=\hat{n} \times\left. H\right|_{S} .
$$

Fig. 4 shows $\mathrm{TE}_{01}$ and $\mathrm{HE}_{11}$ current distribution on the HTS thin film. Current of the $\mathrm{TE}_{01}$ mode is circumferential and, therefore, there is no current flowing from the edge of the HTS film to the enclosure. On the contrary, the $\mathrm{HE}_{11}$ mode has radial currents, and a good contact is needed between the HTS film and the enclosure.

\section{RESONANT FREQUENCY AND UNLOADED $Q$}

Table I shows a comparison between measured and simulated results. Resonant frequencies were obtained from the analysis in Section III, considering no loss in cavity enclosure, dielectric material, and HTS film. Losses were taken into account later through a perturbational analysis that led to the quality factors.
Measurements of the resonator have been performed at 300 and $77 \mathrm{~K}$. Measurements at $77 \mathrm{~K}$ were done in liquid nitrogen and using either silver or HTS film in the cavity bottom plate. At $300 \mathrm{~K}$, we used only silver in the bottom plate of the cavity. A surface resistance of $16 \mu \Omega$ is assumed for the HTS film at $2 \mathrm{GHz}$ and $77 \mathrm{~K}$. The dielectric used is $\mathrm{ZrSnTiO}_{4}$ with a quality factor of $16 \times 10^{3}$ at $300 \mathrm{~K}$. This material doubles its quality factor when cooled to $77 \mathrm{~K}$ [6]. The main loss contribution is due to the dielectric material itself and it highly compromises the overall quality factor of the resonator. The second major loss contribution is the silver-plated enclosure. Losses due to liquid nitrogen can be neglected in the analysis since they are much lower than losses in dielectric material or cavity enclosure. Also, losses in the HTS film can be neglected.

Good agreement is found between theoretical and measured values for $\mathrm{TE}_{01}$ single mode. For $\mathrm{HE}_{11}$ dual mode, however, the measured quality factors were lower than the expected values. This discrepancy could be explained by the existence of radial currents in the $\mathrm{HE}_{11}$ mode. In fact, for the $\mathrm{HE}_{11}$ mode and for the resonator under study, the maximum current on the HTS film is only ten times higher than the maximum radial current at the edge. In our measurements, there was no contact between the film and the enclosure and this can be an explanation for the low measured $Q$ values for $\mathrm{HE}_{11}$ mode.

\section{POWER-HANDLING CAPABILITY}

The high nonlinearity of HTS films is a matter of concern in the image hybrid dielectric/HTS resonator [7]. Measurements performed with a hybrid dielectric/HTS filter show that there is a maximum power that can be handled by the filter [8]. Below this power level, filter performance has a limited but measurable decay. On the contrary, this dependence becomes very strong when the input power exceeds this maximum. Therefore, in order to estimate the power-handling capability, it can be assumed that the quality factor of the resonator is power insensitive.

This power-handling capability is associated with a critical current density above which the RF losses of the HTS film rapidly increase above normal metal losses. At the same time, this critical current density can be associated with a critical magnetic field applied to the surface of the film (2).

For a given input power, the energy stored in the resonator can be calculated from a closed expression [3], [9]. When the resonator is critically coupled, $P_{\text {in }}=P_{\text {diss }}$ and the following equation can be used:

$$
W=\frac{Q}{\omega} P_{\text {in }}
$$

where $Q$ is the quality factor of the resonator, $\omega$ is the resonant frequency, $W$ is the energy stored in the resonator, and $P_{\text {in }}$ is the input power. The scaling is done by noting that the maximum peak value of the magnetic field on the HTS surface is proportional to the energy stored in the resonator, with a proportionality constant which only depends on the mode 
TABLE I

Dielectric Constant 36.3; Resonator Dimensions $1.125 \times 0.506$ in; CaVity Dimensions $2.10 \times 1.034$ in

\begin{tabular}{|c|c|c|c|c|}
\hline & \multicolumn{2}{|c|}{ Measured } & \multicolumn{2}{|c|}{ Computed } \\
\hline Mode and Temperature & $\mathrm{f}(\mathrm{GHz})$ & Q & $\mathrm{f}(\mathrm{GHz})$ & $\mathrm{Q}$ \\
\hline $\mathrm{TE}_{01}$ mode @300K & 2.166 & 9,600 & 2.174 & 8,704 \\
\hline $\begin{array}{l}\mathrm{TE}_{01} \text { mode @77K } \\
\text { without HTS film }\end{array}$ & 2.148 & 20,300 & 2.174 & 18,231 \\
\hline $\begin{array}{l}\mathrm{TE}_{011} \text { mode@77K } \\
\text { with HTS film }\end{array}$ & 2.146 & 31,500 & 2.174 & 28,838 \\
\hline $\mathrm{HE}_{11}$ mode @300K & 1.930 & 4,800 & 1.672 & 5,772 \\
\hline $\begin{array}{l}\mathrm{HE}_{11} \text { mode @77K } \\
\text { without HTS film }\end{array}$ & 1.781 & --- & 1.672 & 12,445 \\
\hline $\begin{array}{l}\mathrm{HE}_{11} \text { mode } @ 77 \mathrm{~K} \\
\text { with HTS film }\end{array}$ & 1.868 & --- & 1.672 & 31,746 \\
\hline
\end{tabular}

TABLE II

Maximum Microwave Magnetic Field on the Surface of THE HTS Film For DifFerent INPUt POWER VAlues

\begin{tabular}{ccccc}
\hline$P_{\text {in }}$ (watts $)$ & 20 & 40 & 60 & 80 \\
\hline $\mathrm{H}_{\max } \mathrm{TE}_{01}(\mathrm{~A} / \mathrm{m})$ & $2.83 \cdot 10^{3}$ & $4.01 \cdot 10^{3}$ & $4.91 \cdot 10^{3}$ & $5.67 \cdot 10^{3}$ \\
$\mathrm{H}_{\max } \mathrm{HE}_{11}(\mathrm{~A} / \mathrm{m})$ & $7.58 \cdot 10^{3}$ & $1.07 \cdot 10^{4}$ & $1.31 \cdot 10^{4}$ & $1.51 \cdot 10^{4}$ \\
\hline
\end{tabular}

considered. Thus, for a given mode

$$
\frac{H_{\max }}{\sqrt{W}}=\frac{H_{\max }^{*}}{\sqrt{W^{*}}}
$$

where $H_{\max }$ is the actual maximum peak value of magnetic field, $H_{\max }^{*}$ the value obtained through numerical calculation, and $W^{*}$ the energy obtained in the numerical analysis. By combining (3) and (4), the maximum magnetic field $H_{\max }$ is calculated for several values of input power (Table II).

Results indicate that $\mathrm{TE}_{01}$ mode can sustain higher power levels than $\mathrm{HE}_{11}$ mode. A value for the critical magnetic field which induces the critical current density in a high-quality YBCO film is found about $1.5 \times 10^{4} \mathrm{~A} / \mathrm{m}$ in [10]. Assuming this critical field value, the dual-mode $\mathrm{HE}_{11}$ resonator starts to fail for an input power of $80 \mathrm{~W}$, whereas the single-mode $\mathrm{TE}_{01}$ resonator can handle up to $560 \mathrm{~W}$. This is in reasonable agreement with measurements performed for the $\mathrm{HE}_{11}$ mode in [8].

\section{CONCLUSIONS}

The hybrid dielectric/HTS resonator has been analyzed using a full-wave radial mode-matching technique. Agreement has been found between measured and simulated results. The method discussed can be used to compute the field or current distributions and therefore, the resonant frequency and quality factor of the resonator. The current distribution on the HTS film for single $\mathrm{TE}_{01}$ and dual $\mathrm{HE}_{11}$ modes has been computed. The existence of radial currents in the $\mathrm{HE}_{11}$ mode requires a good contact between the housing and the film.
Performance of the dielectric/HTS resonator at $77 \mathrm{~K}$ is limited by dielectric material loss. Therefore, optimized materials at cryogenic temperatures, with low loss and high dielectric constant, are needed in order to build competitive resonators and filters.

Finally, the power handling capability of the resonator has been determined. The input power is used to compute the energy stored in the resonator. From this energy value, the maximum peak value of the magnetic field on the surface of the HTS film can be determined as a function of input power. Therefore, if some value of critical field is assumed for the HTS film, the power handling capability of the resonator can be estimated.

\section{REFERENCES}

[1] J. A. Curtis, S. J. Fiedziusko, and S. C. Holme, "Hybrid dielectric/HTS resonators and their applications," IEEE MTT-S Dig., vol. N-2, pp. 447-450, 1991.

[2] R. Mansour, "A C-band superconductive input multiplexer for communication satellites," IEEE Trans. Microwave Theory Tech., vol. 42, no. 12, pp. 2472-2479, Dec. 1994.

[3] N. Tellmann, N. Klein, U. Dähne, A. Scholen, H. Schulz, and H. Chaloupka, "High- $Q \mathrm{LaAlO}_{3}$ dielectric resonator shielded by YBCIfilms," IEEE Trans. Appl. Superconduct., vol. 4, pp. 143-148, Sept. 1994.

[4] D. Kajfez and P. Guillon, Dielectric Resonators. Dedham, MA: Artech House, 1986, pp. 185-209.

[5] S.-W. Chen and K. A. Zaki, "Dielectric ring resonators loaded in waveguide and on substrate," IEEE Trans. Microwave Theory Techn., vol. 39, pp. 2069-2076, Dec. 1991.

[6] H. Tamura, H. Matsumoto, and K. Wakino, "Low temperature properties of dielectric resonator materials," in The 3rd Asia-Pacific Microwave Conf. Proc., Tokyo, Sept. 1990, pp. 1213-1216.

[7] T. Van Duzer and C. W. Turner, Principles of Superconductive Devices and Circuits. New York: Elsevier North-Holland, 1981.

[8] R. R. Mansour, B. Jolley, S. Ye, F. S. Thomson, and V. Dokas, "On the power-handling capability of high-temperature superconductive filters," IEEE Trans. Microwave Theory Tech., vol. 44, pp. 1322-1338, July 1996.

[9] G. Matthaei, L. Young, and E. M. T. Jones, Microwave Filters, Impedance-Matching Networks, and Coupling Structures. Dedham, MA: Artech House, 1980.

[10] C.-W. Lam, D. M. Sheen, S. M. Ali, and D. E. Oates, "Modeling the nonlinearity of superconducting strip transmission lines," IEEE Trans. Appl. Superconduct., vol. 2, pp. 58-66, June 1992. 


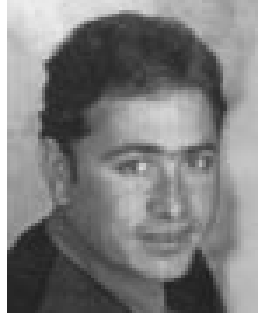

Carles Sans was born on May 14, 1968 in Mataró, Spain. In 1993, he received the Ingeniero (B.S and M.S.) degree in telecommunication engineering from the Polytechnic University of Catalonia (UPC), Barcelona, Spain. Since 1994, he has been working on the Ph.D. degree at the same university.

From 1991 to 1993, he was awarded a scholarship from the University-Industry Foundation to work part-time at Hewlett-Packard Española, S.A. In 1993, he worked on a synthetic aperture radar simulator and processor for satellite applications in the context of the activities of the ESA ERS-1 Fringe Working Group. In 1994 he started a Ph.D. on the application of high-temperature superconductors to communication circuits. He worked on the microwave characterization of high-temperature superconductors and developed several superconducting filters and resonators for low-power applications. From 1995 to 1996, he spent eight months at Conductus, Inc., Sunnyvale, CA, as a Member of the Technical Staff, developing high-power superconducting transmit filters and resonators. His current interests are communication circuits, applied superconductivity, DR cavity filters, and computational electromagnetics.

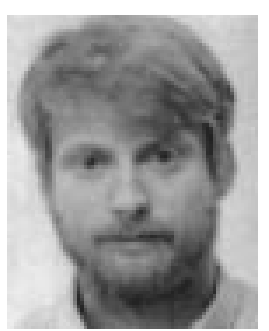

Juan M. O'Callaghan received the telecommunication engineering degree from Universitat Politécnica de Catalunya (UPC), Barcelona, Spain, in 1987 and the M.S. and Ph.D. degrees from the University of Wisconsin, Madison.

He was an intern at Honeywell's Systems Research Center from July to December 1989. In 1993, he joined the faculty at UPC where he has been teaching courses on radar and high frequency devices and systems. His research interest include microwave devices and systems, high frequency transistor characterization, and superconducting devices.

David Sancho received the Ingeniero degree in telecommunications engineering from the Polytechnic University of Catalonia (UPC), Barcelona, Spain, in 1997.

He is currently working at Telefónica Sistemas, Barcelona, Spain.

Rafael Pous (S'89-M'93) was born in Barcelona, Spain, in 1964. He received the Ingeniero degree in telecommunications engineering and the Licenciado degree in computer science from the Polytechnic University of Catalonia (UPC), Barcelona, Spain, in 1988, the M.S. degree in electrical engineering from the University of Massachusetts, Amherst, in 1989, and the Ph.D. degree in electrical engineering from the University of California at Berkeley in 1992.

In 1993, he joined the telecommunications engineering faculty of the Polytechnic University of Catalonia. His research interests are in the area of computational electromagnetics and applied superconductivity.

Dr. Pous was awarded the Fulbright Scholarship and the Schlumberger Fellowship during his studies.

Josep Fontcuberta received the degree in physics from the University of Barcelona, Spain, in 1977 and the Ph.D. degree in physics from the University of Barcelona in 1982.

$\mathrm{He}$ is currently Senior Staff Scientist of the Consejo Superior de Investigaciones Cientificas, Spain, at the Materials Science Institute of Barcelona where he works on superconducting oxides and colossal magnetoresistance prevoskites. His main areas of experience and research include electric and magnetic properties of superconducting and magnetic materials. He has published over 140 regular papers on solid-state physics.

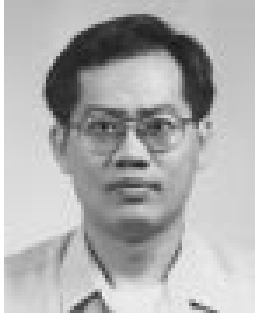

Ji-Fuh Liang was born on November 25, 1958, in Taiwan. He received the B.S. degree in electronics engineering from the National Chiao-Tung University, Taiwan, and the M.S. degree in electrical engineering from the National Taiwan University, Taiwan, in 1981 and 1985, respectively. He received the Ph.D. degree in electrical engineering from the University of Maryland, College Park, in 1994.

From 1981 to 1983 , he was with the Taiwan Military as a Member of the Technical Staff. He spent three years from 1985 to 1988 as a Member of the Technical Staff and Project Leader at Microelectronics Technology, Inc., Hsin-Chu, Taiwan. During these years, he developed MIC components and subsystem integration for microwave digital communication system. $\mathrm{He}$ implemented a cross-coupling and tuning method for $\mathrm{TE}_{01}$ mode $\mathrm{DR}$ cavity filter for Allen Telecom Group during 1994 to 1995 . He worked for Celwave from 1995 to 1996 where he continued to development new types of $\mathrm{TE}_{01}$ mode DR cavity filters, including canonical asymmetric filters, three-pole elliptic function filters, mixed metallic/DR filters, and duplexers. He joined Conductus, Sunnyvale, CA, in 1996 and is in charge of technology development for high-power transmit filters based on hightemperature superconductors. His current interests are superconductor devices in microwave applications, DR cavity filters, and electromagnetic numerical analysis and its applications in microwave components designs, especially for microwave filters.

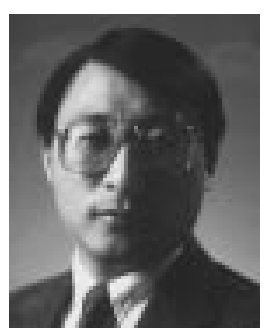

Guo-Chun Liang (S'87-M'90-SM'94) received the B.S. degree from the East China Institute of Technology, Naijing, China, in 1982, the M.S. degree from the University of Electronics Science and Technology of China (UEST), Chengdu, China, 1985, and the Ph.D. degree from University of California at Berkeley, in 1990, all in electrical engineering.

He worked at the microwave center of UEST in 1985-1986. He developed a series of RF and microwave devices, including low noise amplifiers, high power amplifiers, cellular phone package and antenna, attenuators, oscillators, phase shifters, and filters. He also has done a variety of time-domain numerical analyses and simulations of $\mathrm{RF}$ and microwave devices such as transmission lines, amplifiers, antennas, and semiconductor and superconductor devices. He has been with Conductus, Inc., Sunnyvale, CA, since 1990, developing superconductive RF and microwave circuits and systems, including resonators, filters, mixers, delay lines, and magnetic resonance coils. $\mathrm{He}$, with a team at Conductus, has developed space-qualified instantaneous frequency-measurement system for the Navy's High-Temperature Superconductor Space Experiment program (II). He coordinated Conductus's effort in Consortium for Superconducting Electronics activities, collaborating with MIT Lincoln Laboratory, AT\&T, and CTI on high-temperature superconductor wireless product. Currently, he is a Manager in wireless technology leading the development of superconductive circuits and subsystems and cryogenically cooled electronics for wireless communication applications. His interests include RF and microwave applications of superconductivity, microwave circuits and systems, cellular phone applications, numerical analysis, and simulation of practical electromagnetic problems. 